\title{
Corela
}

Cognition, représentation, langage

HS-1 | 2005

Colloque AFLS

\section{Les dérivés de noms propres dans le TLFi : quelles bases pour quels sens?}

\section{Sarah Leroy}

\section{OpenEdition}

\section{Journals}

\section{Édition électronique}

URL : http://journals.openedition.org/corela/1146

DOI : 10.4000/corela. 1146

ISSN : $1638-573 \mathrm{X}$

\section{Éditeur}

Cercle linguistique du Centre et de I'Ouest - CerLICO

\section{Référence électronique}

Sarah Leroy, «Les dérivés de noms propres dans le TLFi : quelles bases pour quels sens ? », Corela [En ligne], HS-1 | 2005, mis en ligne le 16 février 2005, consulté le 30 avril 2019. URL : http:// journals.openedition.org/corela/1146 ; DOI : 10.4000/corela.1146

Ce document a été généré automatiquement le 30 avril 2019

\section{(c) (i) (2)(2)}

Corela - cognition, représentation, langage est mis à disposition selon les termes de la licence Creative Commons Attribution - Pas d'Utilisation Commerciale - Partage dans les Mêmes Conditions 4.0 International. 


\title{
Les dérivés de noms propres dans le TLFi : quelles bases pour quels sens?
}

\author{
Sarah Leroy
}

Ubuesque, chiraquien, marxisme... De nombreux dérivés de noms propres se rencontrent en discours ; certains même, mentionnés dans les dictionnaires, paraissent enregistrés en langue. Or la description du sens de ces dérivés pose problème, dans la mesure où, dans de nombreuses approches, logiques mais aussi linguistiques, le nom propre est considéré comme dépourvu de sens ou muni d'un sens minimal représenté par le prédicat de dénomination être appelé $/ \mathrm{N} /{ }^{1}$.

2 À vrai dire, avant même que ne se pose la question du sens, c'est l'existence même des dérivés de noms propres qui, si l'on s'en tient aux mentions qui en sont faites dans la plupart des manuels de morphologie, est mise en doute ${ }^{2}$. Les linguistes, lorsqu'ils abordent le nom propre du point de vue lexical, ne s'intéressent pas tellement non plus à leur capacité dérivationnelle. On trouve tout au plus quelques paragraphes faisant rapidement un certain nombre d'observations morphologiques (Clarinval 1967: 36-38, Molino 1982: 9-10, Lecomte-Hilmy (1989: 22-25), Jonasson 1994: 34). Il y est généralement posé comme un fait acquis que la productivité morphologique des noms propres est faible, comme l'indique la citation ci-dessous (Molino $1982: 10$ ).

Le nom propre a une possibilité minimale de productivité morphologique (morphologie dérivationnelle). [...] Les noms propres semblent avoir, moins que les noms communs, la possibilité de s'adjoindre préfixes, suffixes ou mots pour engendrer dérivés et composés. [...] Il semble bien que la grande masse des noms propres est peu ou pas productive dans ce domaine.

Les rares dérivés mentionnés sont présentés comme des cas particuliers. Dès lors qu'un sens particulier apparaît, comme dans «donjuanesque, donjuaniser, donjuanisme, donquichottisme, herculéen ou tartu(f)ferie » (Jonasson 1994 : 34), on considère que les noms propres qui en sont la base sont "devenus noms communs» (Molino 1982: 10). Ces (nombreux) cas mis à l'écart, on peut alors lire que la dérivation du nom propre est exceptionnelle et ne concerne pas l'ensemble des noms propres: "notamment les prénoms n'y ont pas accès [...]. Pour la plupart des Npr “ ordinaires ", tels que Paul, Pierre, 
Jean-Jacques, Mireille, Dubos, Martin, Lejeune, etc., qui ne sont associés ni à des personnages politiques importants ou à des écrivains ou des artistes célèbres, ni à des personnages mythologiques ou littéraires exemplifiant un type humain, on ne trouve pas de dérivations [...] » (Jonasson 1994 : 34).

4 L'examen et le relevé des dérivés de noms propres dans un dictionnaire comme le TLFi, ou Trésor de la langue française informatisés ${ }^{3}$ montre le contraire: on y trouve de nombreux dérivés, en particulier suffixaux, de noms propres. Ces dérivés font apparaître une certaine diversité, pour ce qui est de la base sur laquelle ils sont construits (on trouve différents types de noms propres), mais aussi en ce qui concerne les relations sémantiques entre la base et le dérivé.

5 Après avoir décrit les dérivés recensés dans le TLFi, nous les classerons, puis nous aborderons la question des relations sémantiques entre base(s) et dérivé(s).

\section{Les dérivés de noms propres dans le $T L F i$}

6 La recherche menée sur les données du TLFi, bien qu'effectuée manuellement et de façon rudimentaire, en particulier pour ce qui est des comptages $^{4}$, donne des résultats qui entrent en nette contradiction avec le point de vue évoqué ci-dessus, puisqu'elle permet de constituer rapidement une liste d'un millier de dérivés de noms propres, construits avec 32 suffixes différents.

7 Tous ne constituent cependant pas des entrées du TLFi. Au contraire, le statut lexicographique de ces dérivés est variable. Bien sûr, certains constituent parfois des entrées : on trouve par exemple BOUDDHISTE, LIMOGER, ARLEQUINADE, ou même SORBONNARD ; la nature de la base est alors indiquée, de façon plus ou moins directe: on trouve généralement mention du nom propre, sans que soit explicitement indiquée la catégorie grammaticale (ainsi a-t-on, pour moscoutaire, «Dér. de Moscou, nom de la capitale de l'URSS »).

8 Dans la plupart des cas cependant, le dérivé constitue une entrée secondaire au sein d'un article dont l'entrée principale est soit le suffixe concerné (comme goncourable, qu'on trouve dans l'article -ABLE, -IBLE, -UBLE) soit un autre dérivé (on trouve ainsi charentonnais et charentonnade sous l'entrée CHARENTONNESQUE, ou vietnamiser et vietnamisation sous l'entrée VIETNAMIEN).

9 Il peut également arriver qu'un dérivé se trouve dans le TLFi, en quelque sorte, par hasard, dans un exemple retenu pour illustrer un tout autre terme; on trouve ainsi le dérivé dékrouchtchévisation dans l'article DÉDOUANER, dans l'exemple ci-dessous.

Ce film fut pratiquement mis à l'index par Krouchtchev. Dédouané par la dékrouchtchévisation, il marque une étape importante dans le lent dégel soviétique (L'Express, 13 sept. 1965)

10 Les dérivés qui sont ainsi donnés par le biais d'exemples sont souvent, sinon hapaxiques, nettement moins lexicalisés que ceux qui constituent une entrée ou une sous-entrée, leur insertion dans un discours renvoyant à une énonciation donnée.

11 Il arrive parfois qu'un dérivé donné comme sous-entrée soit également présenté au sein d'un exemple. Sous l'entrée -ISSIME, par exemple, on trouve trois dérivés de noms propres, béjartissime, godardissime et josephissime, tous trois introduits par les exemples ci-dessous, 
en une présentation qui semble donc donner, plus que des dérivés lexicalisés, un schéma de construction applicable à tout nom propre.

« Maurice Béjart met en scène «La Tentation de Saint Antoine » de Flaubert [...]. Le spectacle en tout cas s'annonce béjartissime (L'Express, 6 mars 1967, p. 100)».

«Pierrot le Fou : 1965. Il y a le ciel, le soleil et la mer, Karina et Belmondo. Un Godard « godardissime » (L'Express, 15 févr. 1971, p. 6) ».

«Malheureusement, Joseph a été intolérable, ou, pour mieux dire, josephissime. Il

$\mathrm{y}$ a des jours tels, où il semble être la proie du démon joséphique (DUHAMEL,

Maîtres, 1937, p. 46).

Ce dépouillement fait également apparaître la répartition catégorielle des dérivés de noms propres : ceux-ci servent principalement, en termes de nombre de suffixes, de base à des noms communs (16 suffixes, dont -erie, -isme), avec parfois un passage, attesté ou non, par la catégorie verbale (pour les dérivés en -age et en -(is)ation); l'autre grande catégorie est celle des adjectifs (avec 15 suffixes, dont -ard et -esque), avec également un éventuel passage par la catégorie verbale (suffixe -able) et, dans certains cas, possibilité de nominalisation (parisien > un parisien, gaulliste > un gaulliste) ; on trouve enfin des verbes (avec le suffixe -iser) et des noms propres (avec -et(te) et -ot(te), qui construisent également des noms communs). Du point de vue du nombre des dérivés cependant, il est évident que les adjectifs constituent la catégorie la plus importante, avec la très longue liste des adjectifs dits "ethniques $»^{6}$ dérivés de noms de lieux, ou "gentilés» ${ }^{7}$. On constate par ailleurs d'importantes différences de productivité ; certains suffixes, tels que -ais/-ois, -ien ou -iste, montrent nettement des affinités avec le nom propre, alors que pour d'autres (-ité, -ol, -aire), la rencontre paraît accidentelle.

On peut isoler, au sein de cet ensemble de dérivés, un groupe formé par les gentilés et les noms propres (prénoms), caractérisés sémantiquement par le fait que leur interprétation fait fortement intervenir le nom propre de base (on ne peut interpréter maghrébin sans faire appel à un nom propre Maghreb, alors que l'interprétation de figurine peut faire l'économie du retour à figure), et qu'ils sont homogènes du point de vue du type référentiel ${ }^{8}$ (les gentilés se construisent sur des toponymes uniquement, les prénoms sur des prénoms uniquement). Tous les autres dérivés constituent un second groupe, s'alignant sur le fonctionnement sémantique des dérivés de même construction sur base nom commun (à un castriste correspond un partisan de Castro comme à un autonomiste correspond un partisan de l'autonomie) et assez hétérogènes pour ce qui est des types référentiels représentés (bien que les noms de personnes soient majoritaires, on trouve des toponymes, dans moscoutaire ou rouennerie et des prénoms, dans alphonsisme, josephissime ou léonardesque).

On a suggéré ailleurs (Leroy à paraître), que cette différence entre un groupe dérivationnel assez fixe et interne, renvoyant à la forme des noms propres (gentilés et prénoms), et un autre groupe, plutôt libre et ouvert, aligné sur le nom commun, pointant davantage sur le référent habituel ou connu du nom propre (autres dérivés), peut être éclairée par la distinction établie par Gardiner (1954) entre nom propre incarné (embodied ) et désincarné (disembodied), à condition cependant de, à l'instar de Jonasson (1994: 72), ne pas considérer cette distinction comme le classement de deux espèces différentes, mais comme l'expression de deux points de vue différents. En effet, si les noms propres incarnés sont associés de façon stable à un référent particulier (Aristote, Napoléon ou Cicéron), tandis que les noms propres désincarnés existent en tant que formes phoniques et lexicales (Anne, Robert, Duval ou Fabre), reconnues comme des noms propres et stockées comme telles dans la mémoire, il est bien évident que tout nom propre incarné peut 
également être considéré en tant que forme phonique et lexicale, donc désincarné, de même que tout nom propre désincarné peut à tout moment s'incarner.

À partir de là, on voit que les gentilés et prénoms dérivés ont pour base des toponymes et des prénoms désincarnés, considérés avant tout comme des formes phoniques et graphiques de la langue, des unités lexicales, ce qui permet par exemple de créer un gentilé sur n'importe quel toponyme. Inversement, la dérivation du second groupe s'effectue sur des noms propres incarnés, en convoquant le contenu sémantique complet du nom propre (pantagruelesque n'est pas complètement et correctement interprété si le récepteur s'en tient à 'ressemblant à la manière d'être ou d'agir originale d'un individu nommé Pantagruel' : les traits sémantiques convoqués appartiennent au référent du nom propre et participent nécessairement à l'interprétation du dérivé). Le caractère incarné ou désincarné d'un nom propre ne correspondant pas à un statut intrinsèque mais à un point de vue, les deux types de dérivation sont susceptibles de s'effectuer sur chaque base nom propre. Ainsi, Moscou considéré comme désincarné ne pourra servir de base qu'à un gentilé, moscovite, sans autre indication sémantique que 'habitant ou originaire de l'endroit qui a pour nom propre Moscou', tandis que s'il est considéré comme incarné, il pourra donner lieu à d'autres dérivations, sur des bases sémantiques plus riches. La paraphrase donnée pour moscovite, 'habitant ou originaire de l'endroit qui a pour nom propre Moscou', ne convoque du nom propre qu'un sens de type instructionnel ou procédural, le sens de dénomination'. Celle qu'on peut évoquer pour moscoutaire, ronsardiser ou chiraquisme fait, elle, plutôt appel à un sens de type descriptif. C'est ce sens descriptif, qu'on peut rapprocher du contenu proposé par Gary-Prieur (1994:46 et 48), qui sera convoqué dans la plupart des dérivés de nom propre autres que les gentilés et les prénoms.

\section{Les dérivés de noms propres incarnés da ns le TLFi}

Si l'on considère comme "réglé», par l'évocation du seul sens instructionnel de dénomination de la base, le cas des dérivés de noms propres du TLFi construits sur une base désincarnée, on peut désormais évacuer de notre étude quatre suffixes, qui ne donnent lieu, dans le TLFi, qu'à des gentilés : -ais/-ois, -an, -eau et -on. On peut également écarter partiellement trois suffixes qui construisent des gentilés et/ou des prénoms, mais pas uniquement: -ain, essentiellement dédié aux gentilés (africain, toulousain) mais donnant aussi dominicain, élisabéthain, franciscain et olivétain ; - -al, qui donne provençal mais aussi marial ; -in, suffixant, sur un nom propre, des adjectifs ethniques, mais qu'on trouve également dans bénédictin, bernardin, jacobin, manuélin, ursuline. Trois suffixes évaluatifs donnent des dérivés variés : -ette, outre les prénoms, donne catherinette et botticellinette; ot(te), produit des prénoms, un gentilé (solognot) et les noms marengotte et marotte; -ard enfin, produit, en proportions comparables, des gentilés et les adjectifs bondieusard, (anti)dreyfusard, cyrard, napoléonard, philippard, polognard, sorbonnard. Seuls les dérivés sur base incarnée de ces deux derniers groupes de suffixes seront dorénavant considérés, tout comme on ne retiendra pas, de la longue liste des dérivés en -ien,les gentilés.

Parmi les nombreux mots construits avec les quelque 25 suffixes restant, on trouve des choses aussi différentes que ampérage et turlupinage, jacquerie et micheletterie, (le) taylorisme et (un) rousseauisme, des suffixes rares comme dans zinnia, napoléonide, vulcanales ou sternbergite, à ne pas confondre avec wagnerite, et des dérivations 
surprenantes comme celles de bouddhéité ou de cicéronage. La relative hétérogénéité des dérivés amène à relever plusieurs problèmes :

i) Alors que dans la plupart des cas, l'instruction sémantique du suffixe est la même pour une base nom commun et pour une base nom propre, même pour un suffixe aussi « neutre, non marqué » que «-ien: en relation avec le nom base (privilégiant des relations d'origine ou d'appartenance » (Lignon 2002 : 137), qui donne un massif corallien (un massif de corail) comme le style balzacien (le style de Balzac), il arrive que la correspondance sémantique avec un dérivé à base nom commun ne soit pas tout à fait exacte. Ainsi, être goncourable ne signifie pas être susceptible de devenir Goncourt, comme être ministrable signifie être susceptible de devenir ministre, mais, bien sûr, de recevoir le prix Goncourt. De même, si bougainvillier s'aligne sur les noms d'arbres comme pommier ou amandier, la base ne désigne pas le fruit comme c'est habituellement le cas, et dinandier s'éloigne des «noms de personne exer[çant] une activité à caractère professionnel » (TLFi) en ce qu'il n'indique pas la fabrication (dentellière), vente (harengère) ou gestion (hôtelier) du référent désigné par la base (Dinant) mais qu'il a une activité en relation avec le cuivre dont cette ville s'est fait spécialité.

ii) Quelques dérivés de noms propres semblent passer outre les contraintes catégorielles. Si goncourable et académisable s'inscrivent dans le groupe, certes réduit, des dérivés en able sur base nominale (charitable, papable), la suffixation en -ité du nom propre Bouddha ( bouddhéité) constitue une réelle exception; de même, on considère habituellement que le suffixe -issime prend pour base des adjectifs (richissime) et non des noms, même si l'article du TLFi sépare, de façon un peu artificielle, les dérivés à base adjectivale de ceux qui ont pour base un nom propre.

iii) Dans un certain nombre de cas, le lien avec la base nom propre semble être perdu ou du moins fortement atténué : soit le locuteur n'a plus du tout conscience qu'il a affaire à un dérivé de nom propre (ampérage, rocambolesque, dahlia, machiavélique, pasteuriser...) soit, comme dantesque ou ubuesque, "simples appréciatifs disant le caractère excessif et susceptibles de s'appliquer très largement à tout type de référent » (Noailly $2003: 123$ ) ou comme sadique, masochisme, épicurien ou gaulliste, dont le nom propre base n'est pas tout à fait oublié, les dérivés sont dotés d'un sens conceptuel qui permet de faire l'économie d'un passage interprétatif par le nom propre et son référent. On se trouve alors devant ce qu'on qualifie souvent de " lexicalisation » plus ou moins avancée.

21 Outre ces problèmes particuliers, qui concernent tel ou tel dérivé sans distinction catégorielle, d'autres caractéristiques sémantiques se dessinent en fonction de la recatégorisation effectuée par la suffixation.

\subsection{Dérivés adjectivaux}

Les dérivés adjectivaux ${ }^{10}$, qui représentent près de la moitié des dérivés hors gentilés et prénoms, ne se laissent pas toujours répartir suivant la traditionnelle distinction en adjectifs qualificatifs et relationnels. Le cas des suffixés en -issime, qui sont clairement qualificatifs, et de marial, tout aussi clairement relationnel, mis à part, on a, pour les suffixés en -esque, -ien, -ique et -iste, une structure plutôt relationnelle, mais qui devient facilement qualifiante ${ }^{11}$. On assiste en fait, pour -ien ${ }^{12}$ et -iste, à la coexistence des deux emplois : si une victime stalinienne est une victime de Staline, une avenue stalinienne n'est pas une avenue de Staline, mais une avenue qui rappelle, au niveau de l'architecture, de 
l'ambiance, etc. l'époque de Staline. Les exemples ci-dessous font apparaître cette différence.

Cette avenue est stalinienne.

*Cette victime est stalinienne.

De même, l'adjectif, dans le programme poujadiste, est relationnel, tandis qu'il est qualificatif dans l'épicier poujadiste. Moins polyvalent, -esque, tout en gardant la possibilité d'un emploi relationnel (dans le premier des exemples ci-dessous) est plutôt qualificatif (dans le second des exemples ci-dessous) ${ }^{13}$.

Je m'étonne qu'ils [Louis Martin et Henry Bérenger] aient essayé, comme auteur et rapporteur, de nous faire tomber dans cette proposition panthéonesque [le transfert de Diderot]. (BARRÈs, Maîtres, 1923, p.175)

Rien n'était plus rembranesque que cette silhouette de jeune fille, toute dans l'ombre, la plus gracieuse tache qui puisse être faite sur un fond tout de feu et de

lumière. (GONCOURT, Journal, 1862, p. 1083).

Enfin, -ique, plus guère productif, donne des adjectifs presque totalement qualificatifs :

Tu es de plus en plus machiavélique.

Or si les emplois relationnels renvoient au référent du nom propre, c'est-à-dire que le nom propre y retrouve ici son statut d'étiquette pragmatique, de désignateur d'un individu, les emplois qualificatifs font appel à des propriétés plus ou moins typiques du référent, au contenu du nom propre: si le programme poujadiste fait référence à la connaissance encyclopédique du référent, l'épicier poujadiste fait appel à bien d'autres types de propriétés et de connaissances à propos dudit référent.

Ce flottement ne vaut pas pour d'autres suffixes, comme ceux qui permettent de construire des noms de congrégations:- ain (une sœur franciscaine) et -in (un moine bénédictin). S'ils restent nettement relationnels, ces adjectifs ne renvoient pas au référent du nom propre mais à la congrégation à laquelle il a donné son nom. Il y a donc un décalage avec le porteur initial du nom propre, et une substantivation bien établie, tout comme dans le cas du suffixe -ard. Ce dernier semble tantôt relationnel (un étudiant sorbonnard), tantôt qualificatif (Être de plus en plus dreyfusard), et finalement plutôt substantif (un sorbonnard, un dreyfusard). Les dérivés en -able et -aire, quasi hapaxiques ( goncourable, moscoutaire), ne renvoient pas non plus directement à l'individu désigné par le nom propre, mais à un référent voisin ou au contenu du nom propre.

\subsection{Dérivés nominaux et verbaux}

Les dérivés nominaux et verbaux, relativement peu nombreux vis-à-vis des dérivés adjectivaux, sont assez hétérogènes.

Se distingue tout d'abord un groupe constitué de dérivés construits avec des suffixes savants et spécialisés dans telle ou telle terminologie. On trouve ainsi des dérivés relevant $\mathrm{du}$ domaine des sciences naturelles, horticulture (suffixe $-i a^{14}$ ), physique (suffixes $-i e^{15}$ et -ium ${ }^{16}$ ), chimie (suffixe $-\mathrm{ol}^{17}$ ). Les dérivés, noms de plantes (bégonia, magnolia), roches ( berkélium, einsteinium), métaux (sternbergite) sont interprétables sans aucun recours au référent du nom propre qui constitue leur base. On peut donc penser qu'on retrouve ici le fonctionnement des dérivés sur base désincarnée, à ceci près que le sens instructionnel dénominatif n'est même pas convoqué : contrairement à l'interprétation du gentilé alésien , « habitant d'un lieu qui a pour nom Alès », celle de magnolia ne nécessite pas le passage par le nom propre Magnol, qui est généralement tout à fait oublié. 

du littéraire ou du religieux (-ade ${ }^{18}$, -ales ${ }^{19}$ et $\left.-i d e^{20}\right)$, qui sont repris tels quels en français ( bacchanales, noachides $)^{21}$. Il y a alors renvoi au référent du nom propre, de façon directe, comme dans le cas des adjectifs relationnels (les abbassides sont les descendants de Abbas, les saturnales les fêtes de Saturne). Il peut aussi se produire, avec ces suffixes, un phénomène de dérivation par analogie : sont ainsi créés, sur le modèle de Iliade, noachides et bacchanales, les dérivés plus modernes Henriade, napoléonide et mariales; de la même manière, le suffixe -ite dans son acception médicale ${ }^{22}$ permet de construire des dérivés plaisants indiquant un goût immodéré, une admiration excessive, une manie, pour le nom de base (qui peut très bien être un nom propre) : baudelairite, napoléonite; ce n'est alors pas le référent du nom propre lui-même, mais généralement son œuvre, qui est l'objet de la passion.

Les noms dénominaux construits sur la dizaine de suffixes restant, pour la plupart " "appellatifs" ou "dénominatifs" ", servant "à "donner un nom" à une classe d'objets " et mettant "directement le dérivé en relation avec le monde extérieur " $^{23}$ (Roché 2003: 84), tout comme la cinquantaine de verbes dénominaux du corpus, se caractérisent par un décalage interprétatif avec le référent du nom propre. Il n'y a pas, comme avec certains adjectifs, renvoi pur et simple au référent et aux connaissances qu'on peut en avoir, mais plutôt appel à des traits associés au nom propre, parfois intimement parfois de beaucoup plus loin. Ainsi, si patelinage a le sens de «fausseté, intention hypocrite et doucereuse ", en relation avec le contenu de ce nom propre associé au personnage de maître Patelin, si tartufferie signifie "comportement hypocrite » et renvoie au contenu très connu du nom propre Tartuffe, ampérage, et catherinette nécessitent, pour être interprétés en fonction du nom propre base (ce qui n'est en général pas le cas), un détour par une relation métonymique inventeur/inventé (pour ampérage) ou patronne/protégée (pour catherinette).

31 La relation métonymique est clairement présente dans bien des cas, de même que la relation métaphorique, qui est évidente dans les dérivés verbaux : bovaryser n'est bien sûr pas "se transformer en Mme Bovary " mais «se comporter comme Mme Bovary», de même que cicéroniser ne signifie pas «(faire) devenir Cicéron» mais "parler comme Cicéron». Ces relations tropaïques, qu'on trouve aussi avec les adjectifs, ne se répartissent pas selon les suffixes : -isation indique aussi bien des relations métaphoriques (balkanisation) que métonymiques (taylorisation).

\section{Relations tropaïques et dérivation}

32 La situation des dérivés de nom propre est donc assez compliquée : les différents niveaux de sens du nom propre, sens instructionnel de dénomination et sens descriptif ou contenu, interviennent dans l'interprétation, fort différente, des dérivés sur base désincarnée ou incarnée. Mais à ceci vient s'ajouter le fait, fort banal, que la base comme le dérivé suffixal peuvent faire l'objet d'un changement sémantique, de ce qu'on appelle traditionnellement un "transfert de sens", que Roché (2003: 87) préfère nommer "dérivation sémantique », où les figures, et plus précisément les tropes, jouent un grand rôle. La dimension figurale vient donc encore compliquer les choses.

La dérivation sémantique est évoquée par Roché $(2003: 87)$ pour traiter la question de la polysémie des dérivés. Il montre ainsi que bourse ayant deux principaux sens, on peut 
représenter le sens second, métonymique, comme une nouvelle unité lexicale. On a donc bourse $_{1}$ (petit sac destiné à contenir de l'argent), sur lequel est dérivé boursier (fabricant de bourses) et bourse ${ }_{2}$ (somme d'argent), sur lequel est dérivé boursier ${ }_{2}$ (bénéficiaire d'une somme d'argent). Si on admet cette description, on peut parfois considérer qu'on a des dérivations sur des bases non pas $\mathrm{Npr}_{1}$ mais $\mathrm{Npr}_{2}$, ce qui se passe par exemple avec Goncourt $_{1}>$ goncouresque et Goncourt $_{2}$ (le prix littéraire, par métonymie) > goncourable.

\subsection{Procédés métonymiques affectant la base}

Il est alors nécessaire de faire intervenir une étape métonymique affectant la base pour rendre compte de ce qui revient finalement à une double opération de dérivation, qu'on représente par le schéma ci-dessous :

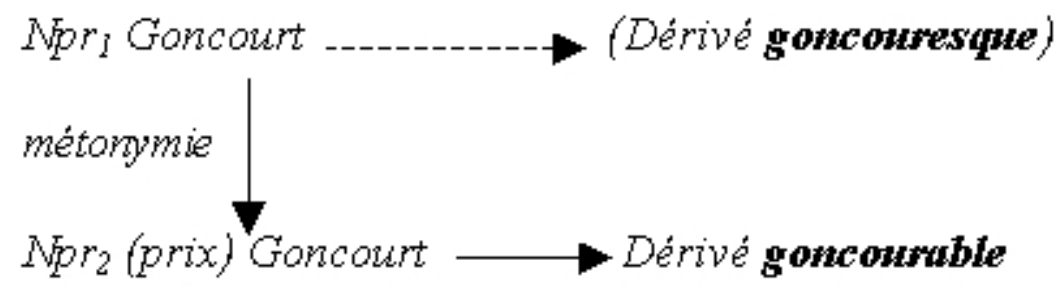

Figure $n^{\circ} 1^{24}$

Cette double possibilité est finalement assez proche de ce qu'on rencontre lorsqu'un même nom propre, tantôt incarné, tantôt désincarné, constitue la base de deux dérivés différents :

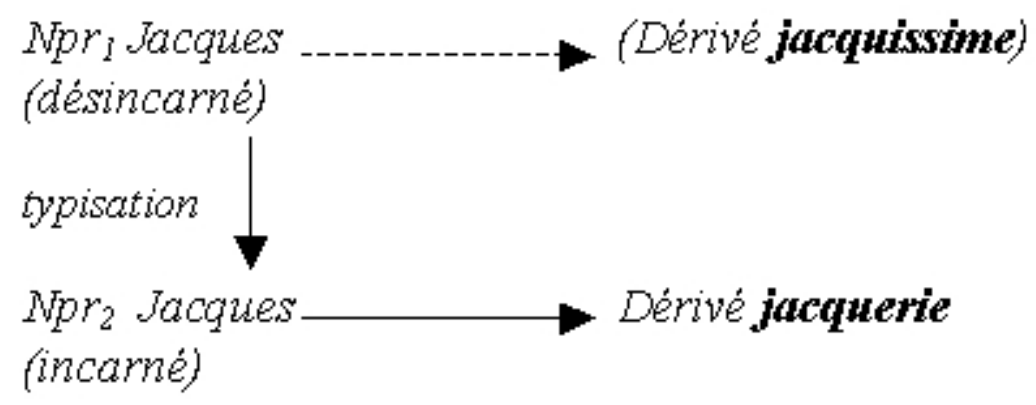

Figure $n^{\circ} 2$

Le dérivé dominicain, également construit sur la base incarnée (Saint) Dominique, relève du même schéma.

L'étape de dérivation métonymique de la base rejoint l'idée de lexicalisation dont il a été question précédemment, à ceci près que la dérivation sémantique n'entraîne pas automatiquement un changement de catégorie. Certes, ampérage est dérivé de Ampère ${ }_{2}$, devenu nom commun à la suite d'une métonymie, mais on ne peut pas étendre ce changement de catégorie à tous les cas: le Goncourt de goncourable, par exemple,n'y répond pas. Le schéma de dérivation de ampérage serait donc une variante de celui de goncourable. 


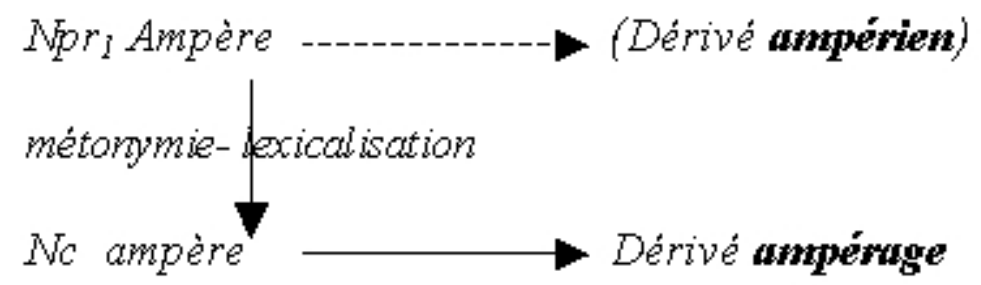

Figure $n^{\circ} 3$

Cette dérivation sur base métonymique peut aussi se faire en " économisant » cette base seconde, comme c'est le cas par exemple pour pasteuriser, qui construit directement un dérivé métonymique fort différent d'un possible dérivé direct pasteurien, sans pour autant qu'une base Pasteur ${ }_{2}$ soit envisageable. Dérivation suffixale et dérivation sémantique se font alors dans un même mouvement :

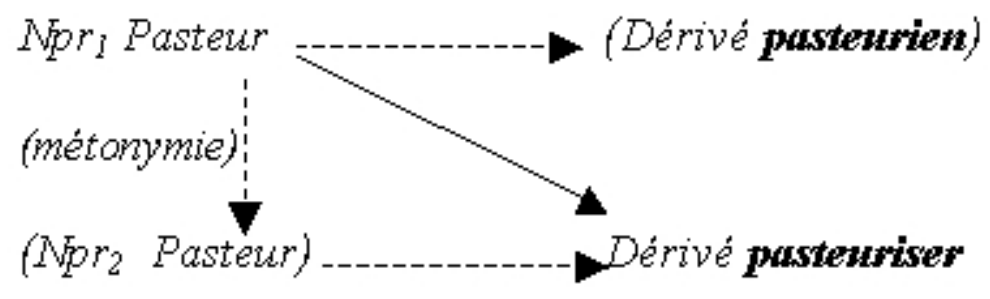

Figure $n^{\circ} 4$

\subsection{Procédés métaphoriques affectant le dérivé}

Dans d'autres cas, c'est sur le dérivé suffixal que s'effectue la dérivation sémantique : si une mazarinade est au départ une satire contre Mazarin (donc mazarinade < Mazarin), le terme désigne, dans une " extension de sens » (TLFi) toute attaque, toute satire. On a donc un seul Mazarin, mais deux mazarinade, le second étant sémantiquement dérivé du premier par métaphore.

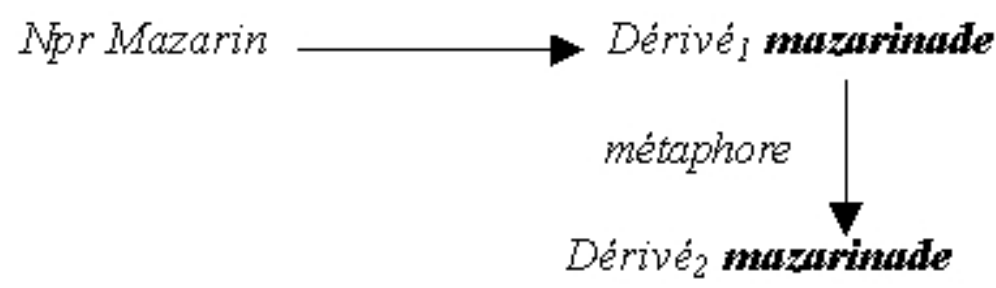

Figure $n^{\circ} 5$

Les choses sont encore plus claires lorsque apparaissent nettement deux niveaux d'interprétation du nom propre, selon que celui-ci est considéré comme simple désignateur rigide ou du point de vue de son contenu. C'est une situation qu'on peut illustrer avec le cas de dantesque: comme le montre très bien l'article du TLFi, il n'y a qu'une base, le Npr Dante, pour deux acceptions de ce dérivé, dantesque, " propre à la poésie de Dante " (adjectif relationnel, où le nom propre a son statut traditionnel de désignateur) et dantesque ${ }_{2}$, " qui imite ou rappelle le caractère (terrifiant, grandiose etc.) 
de la Divine Comédie de Dante » (adjectif qualificatif, où c'est le contenu du nom propre qui est exploité). On voit bien alors que la relation du second dérivé avec le nom propre de base n'est pas perdue: ce dérivé exprime aussi une relation métaphorique avec le référent du nom propre, ce que tente de représenter la flèche transversale :

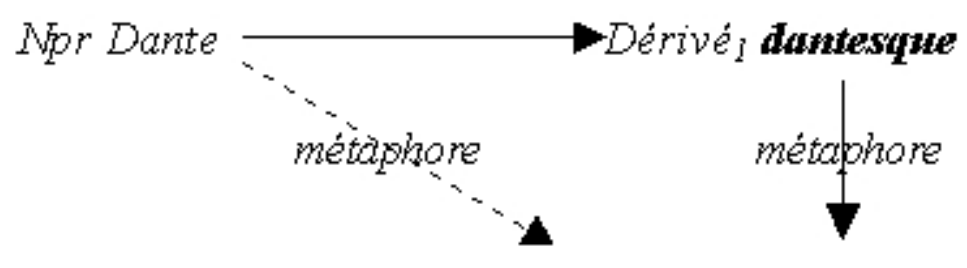

Dérivé 2 dantesque

Figure $n^{\circ} 6$

Il arrive parfois que cette différence entre les niveaux d'accessibilité du référent du nom propre soit marquée par l'emploi de suffixes différents: on ainsi sadien et marxien, relationnels et concernant directement le référent, et sadique et marxiste, concernant le référent. Il n'y a alors plus de relation entre les différents dérivés, mais deux dérivations sur des aspects sémantiques différents, constituant des relations dérivationnelles distinctes.

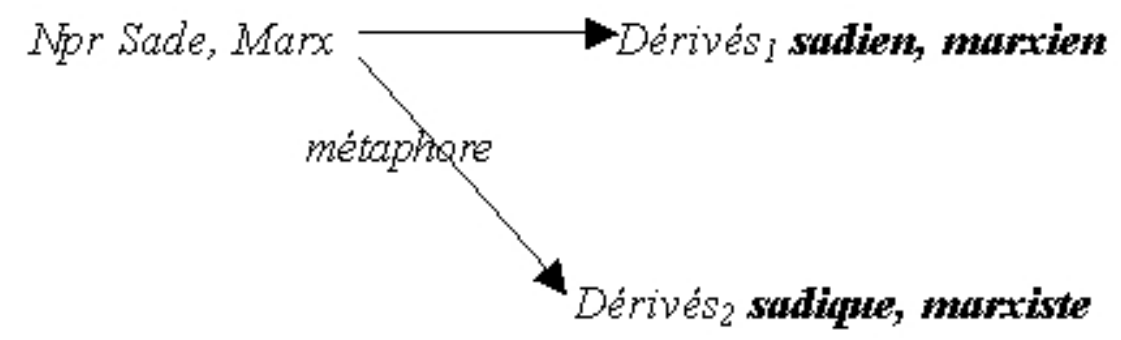

Figure $n^{\circ} 7$

42 On rencontre, dans ce type de dérivation également, des cas de lexicalisation : c'est ce qui se passe lorsque le nom propre d'origine est finalement oublié et qu'un sens conceptuel vient s'associer de façon stable au dérivé, le retour interprétatif vers le nom propre devenant inutile. On peut considérer que c'est ce qui se passe lorsqu'un dérivé ${ }_{1}$ est oublié, sort de l'usage, tandis qu'un dérivé ${ }_{2}$ subsiste, ce que tentent de représenter les schémas ci-dessous.

Ainsi, l'adjectif rocambolesque, dans son premier sens, relationnel, se rapporte directement au référent du nom propre Rocambole «nom du héros aux aventures extraordinaires des romans-feuilletons de P.-A. Ponson du Terrail, parus à partir de 1859 » (TLFi); ce sens relationnel n'est pour ainsi dire jamais exploité, le sens «actif» de l'adjectif étant son sens qualificatif, "invraisemblable, plein de péripéties extraordinaires» (TLFi). La relation métaphorique entre les deux dérivés étant perdue, l'origine propriale du dérivé s'estompe. 


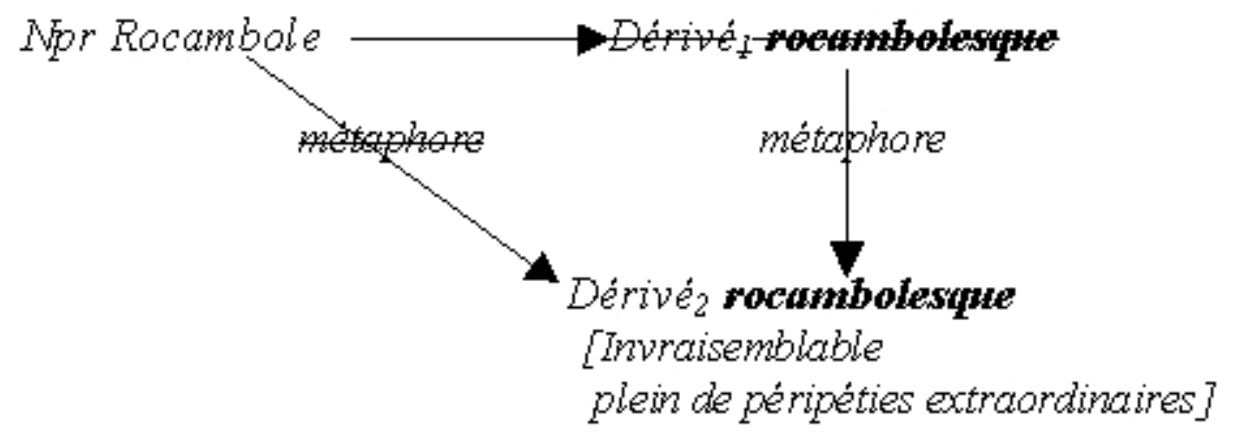

Figure $n^{\circ} 8$

On peut proposer une analyse identique de machiavélique qui, comme beaucoup de dérivés en -ique, est fortement lexicalisé. Le premier dérivé est relationnel, comme l'indique la définition du TLFi : "conforme à la doctrine politique de Machiavel considérée comme dépourvue de tout sens moral, d'honnêteté et d'intégrité ». Si cet adjectif peut toujours être employé ainsi, le sens le plus répandu est cependant celui du dérivé ${ }_{2}$, "qui est conçu, réalisé dans un esprit de perfidie, de mauvaise foi et de déloyauté » (TLFi), et la relation sémantique métaphorique entre ce second dérivé et le nom propre initial semble effacée.

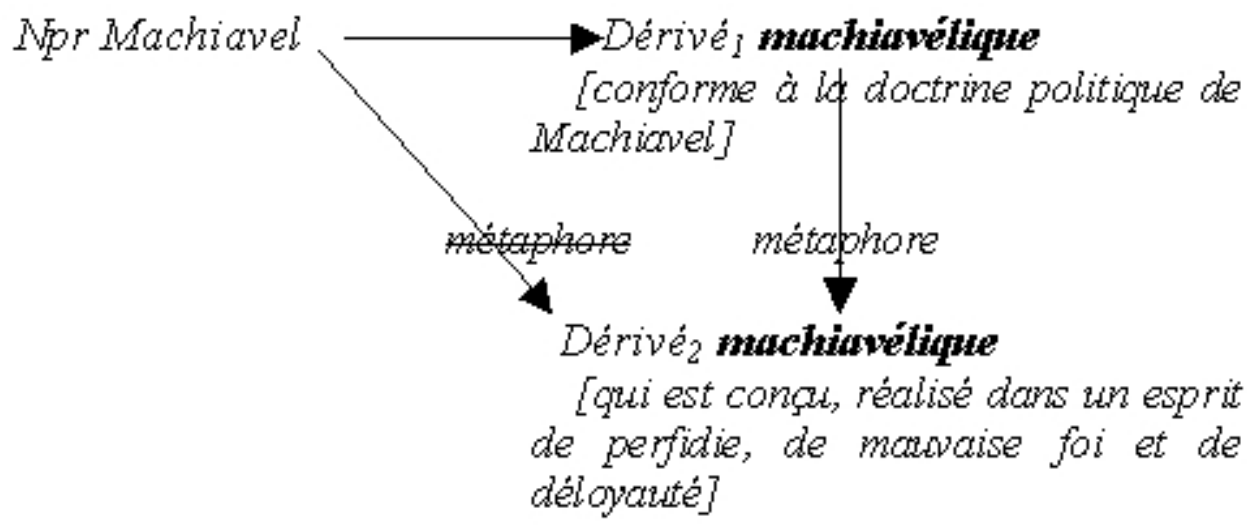

Figure $n^{\circ} 9$

\section{Conclusion}

L'exploration des données du TLFi permet donc de «découvrir " l'importance de la dérivation des noms propres, mais surtout sa diversité. L'hétérogénéité du corpus de dérivés constitué est en partie surmontée grâce aux apports théoriques des travaux de linguistique du nom propre, mais de nombreux cas restent difficiles à intégrer aux schémas dérivationnels habituellement associés à tels ou tels affixes. Un détour par la dérivation sémantique, qui s'appuie sur des opérations tropaïques de type métonymique ou métaphorique se révèle nécéssaire pour décrire l'écart qui s'établit, dans le cas des noms prorpes plus qu'ailleurs, entre base et dérivé. 


\section{BIBLIOGRAPHIE}

BARTNING, I., 1986, « Le parallélisme entre les syntagmes Nom + Adjectif ethnique et les syntagmes prépositionnels correspondants en Nom + de + (+ Det)+Nom géographique », Revue romane 21-1, 3-52 BARTNING, I. et NOAILLY, M., 1993, « Du relationnel au qualificatif : flux et reflux », L'Information grammaticale $58,27-32$

BARTNING, I. et NOAILly, M., 1995, « Pourquoi-esque ? », Cahiers de grammaire 20, 87-100

BONNET, V., 2003, « Pour une terminologie diachronique », Travaux linguistiques du CerLiCO 16, 27-47

BÜCHI, E., 1996, Les Structures du « Französiches Etymologisches Wöterbuch ». Recherches métalexicographiques et métalexicologiques, Tübingen : Niemeyer

CLARINVAL, B., 1967, « Essai sur le statut linguistique du nom propre », Cahiers de lexicologie 11, 24-44

GARDINER A., 1954, The Theory of Proper Names. A Controversial Essay, Londres : Oxford University Press

JONASSON, K., 1994, Le Nom propre. Constructions et interprétations, Louvain-la-Neuve : Duculot KLEIBER, G., 1981, Problèmes de référence : descriptions définies et noms propres, Klincksieck

KLEIBER, G., 1996, « Noms propres et noms communs : un problème de dénomination », Meta 41-4, 567-589

LECOMTE-HILMY, A., 1989, « Du statut linguistique des noms propres dans cinq dictionnaires français ", Cahiers de lexicologie 54, 7-32

LEHMAN, A. et MARTIN-BERTHET, F., 2000, Introduction à la lexicologie. Sémantique et morphologie, Nathan

LEROY S., (à paraître), « Les noms propres et la dérivation suffixale », Meta

LIGNON, S., 2000, La Suffixation en -ien. Aspects sémantiques et phonologiques, thèse de doctorat, Université Toulouse-Le Mirail, Toulouse II

LIGNON, S., 2002, « L'adjectif en -ien comme révélateur de phénomènes de concurrence », BULAG

$27,135-150$

MOLINO, J., 1982, «Le nom propre dans la langue » Langages 66, 5-20

NOAILLY, M., 2003, « Malice et mise en discours : quelques données nouvelles sur-esque », Travaux linguistiques du CerLiCO 16, 119-127

PR1 : REY-DEBOVE, J. et REY, A., 1993 [2000], Le Nouveau Petit Robert. Dictionnaire alphabétique et analogique de la langue française, Paris : Dictionnaires Le Robert (édition remaniée et amplifiée de Robert, P., 1967, Le Petit Robert. Dictionnaire de la langue française, Paris : Le Robert)

ROCHÉ, M., 2003, « Catégorisation et recatégorisation en morphologie dérivationnelle : le cas de la dérivation en -ier(e) », Travaux linguistiques du CerLiCO 16, 75-92 


\section{ANNEXES}

\section{Tableau des suffixes}

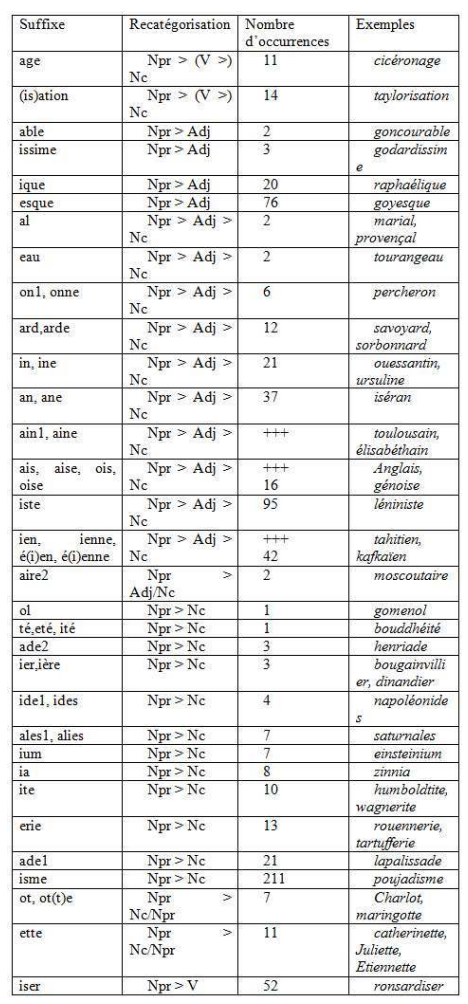

\section{NOTES}

1. Kleiber (1981:331).

2. Ainsi, Lehman et Martin-Berthet (2000:5) éliminent les noms propres dès l'abord, les situant, avec « les mots “virtuels" » et « les mots étrangers », « aux frontières du lexique »: « ils ne sont pas des mots de la langue, parce qu'ils n'ont pas de sens ».

3. ATILF (Analyse et Traitement Informatique de la Langue Française) et Université Nancy II, http:// zeus.inalf.cnrs.fr/.

4. Les chiffres du tableau récapitulatif donné en annexe doivent être considérés avec prudence, mais donnent néanmoins un ordre de grandeur des phénomènes. Pour certains suffixes très productifs, on se contente du symbole ${ }^{+++}$, qui indique un nombre élevé d'occurrences.

5. Büchi (1996 : 290) appelle ce commentaire étymologique « glose linguistico-référentielle ».

6. Terme de Bartning (1986).

7. La « liste des noms communs et des adjectifs correspondant aux noms propres de personnes et de lieux » du PR1 (2000: 2727-2739), nullement exhaustive, donne ainsi 1807 adjectifs dérivés de noms propres, dont 1509 toponymiques.

8. C'est-à-dire du type de référent auquel renvoie le nom propre (toponyme, anthroponyme, etc.).

9. Cf. Kleiber (1996).

10. Dont de nombreux peuvent être substantivés.

11. Ce qui n'a en soi rien d'exceptionnel. Cf. Bartning et Noailly (1993). 
12. Cf. Lignon (2000).

13. Cf. Bartning et Noailly (1995) et Noailly (2003).

14. "Suff. sav. entrant dans la constr. de subst. masc. désignant des fleurs et des arbres ornementaux» (TLFi).

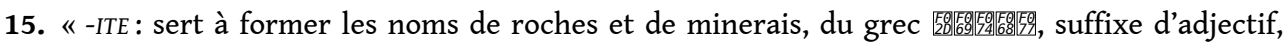

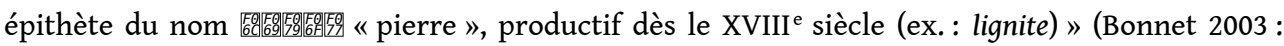
29).

16. «-IUM : utilisé depuis la fin du XVIII ${ }^{\mathrm{e}}$ siècle pour désigner les métaux ou les métallö̈des, vient de la terminaison -ium de certains noms de métaux dans le latin classique » (Bonnet $2003: 38$ ).

17. «-OL: signifie « huile », du morphème olé $(o, i)$, du latin oleum, utilisé à partir de 1832 ; plus ou moins systématisé en 1840 par Gerhardt pour désigner les huiles (ex. : furfurol)» (Bonnet 2003 : 29).

18. "Suff. sav. servant à former des subst. fém. exprimant la notion d'ensemble ou d'élément d'un groupe » (TLFi).

19. «Suff. formateur de subst. fém. plur. désignant des fêtes dans l'Antiq. Romaine [...] Les subst. en -ales sont tous empruntés du lat. » (TLFi).

20. «Suff. patronymique issu du gr. [éventuellement] par l'intermédiaire du lat. -is, -idis formateur de subst. " (TLFi).

21. On peut ajouter catilinaire à cette section.

22. Alors «les mots constr. sont des subst. fém. désignant des affections de nature inflammatoire » (TLFi).

23. A la différence d'autres suffixes qui permettent «d'appréhender autrement la catégorie dénotée par le mot base » (Roché $2003: 84$ ).

24. Les parenthèses et la flèche en pointillés indiquent ici que ces dérivés sont possibles mais non attestés.

\section{RÉSUMÉS}

Alors que la dérivation du nom propre est généralement considérée comme faible, voire nulle, l'examen des données du TLFi permet de constituer un corpus de dérivés important mais hétérogène. La description et l'analyse de ces données nous conduit à en proposer un classement fondé sur le caractère incarné ou désincarné du nom propre de base puis, pour les dérivés sur base incarnée, sur les relations sémantiques établies entre base et dérivé. Dans bon nombre de cas, les écarts de sens constatés s'expliquent par des relations tropaïques qui interviennent sur l'un ou l'autre élément de la dérivation.

Even though proper name derivation is mostly regarded as weak, even null, the scan of TLFi data leads to an important - although heterogeneous - corpus of derivatives. From the description and analysis of these data, we propose a classification, built as follows : first, on the main character of the basic proper name (embodied or disembodied) ; then, for embodied based derivatives, on the semantic relationships established between the basis and the derivative. In numerous cases, the meaning variations can be explained by tropaïc relationships which apply on each element of the derivation. 
INDEX

Mots-clés : dérivation, dictionnaire, morphologie

\section{AUTEUR}

SARAH LEROY

UMR CNRS 7114 (Université Paris X-Nanterre) 